Molecules 2000, 5, 714-726

\title{
molecules
}

ISSN 1420-3049

http://www.mdpi.org

\section{Synthesis of N,N'-Diarylalkanediamides and Their Antimycobacterial and Antialgal Activity}

\author{
Lenka Kubicova $^{1}{ }^{*}$, Karel Waisser ${ }^{1}$, Jiri Kunes ${ }^{2}$, Katarina Kralova $^{3}$, Zelmira Odlerova ${ }^{4}$, Milan \\ Slosarek $^{5}$, Jiri Janota ${ }^{5}$ and Zbynek Svoboda ${ }^{6}$
}

${ }^{1}$ Department of Inorganic and Organic Chemistry, Charles University in Prague, Faculty of Pharmacy in Hradec Kralove, Heyrovskeho 1203, 50005 Hradec Kralove, Czech Republic

Tel.: +420 49 5067339, Fax: +420 49 5210002, E-mail: kubicova@faf.cuni.cz

${ }^{2}$ Laboratory of Structure and Interactions of Biologically Active Molecules, Charles University in Prague, Faculty of Pharmacy in Hradec Kralove, Heyrovskeho 1203, 50005 Hradec Kralove, Czech

Republic

${ }^{3}$ Institute of Chemistry, Faculty of Natural Sciences, Comenius University, Mlynska dolina CH-2, 842

15 Bratislava, Slovak Republic

${ }^{4}$ Institute of Preventive and Clinic Medicine, Limbova 14, 83501 Bratislava, Slovak Republic

${ }^{5}$ National Institute of Public Health, Srobarova 48, 10042 Praha 10, Czech Republic

${ }^{6}$ Institute of Experimental Biopharmaceutics, Heyrovskeho 1207, 50002 Hradec Kralove, Czech

Republic

Received: 2 November 1999 / Accepted: 13 December 1999 / Published: 14 May 2000

\begin{abstract}
A set of N,N'-diarylalkanediamides was synthesized. The compounds were tested for their antimycobacterial and antialgal activity. The antimycobacterial activity of N,N'diarylalkanediamides depends on the lipophilicity of the respective acid. Antimycobacterially active substances were found only in the series of N,N'-diarylethanediamides and N,N'diarylbutanediamides. Other compounds (derivatives of pentane-, hexane-, octane- and nonanediamide) were inactive against various strains of mycobacteria. The compounds inhibited growth and chlorophyll production in Chlorella vulgaris. Their relatively low antialgal activity is probably connected with their lowered aqueous solubility, and hence by a restricted passage of the inhibitor through the hydrophilic regions of thylakoid membranes.
\end{abstract}

Keywords: N,N'-diarylalkanediamides, antimycobacterial activity, antialgal activity.

(C) 2000 by MDPI (http://www.mdpi.org). Reproduction is permitted for noncommercial purposes. 


\section{Introduction}

As the end of the $20^{\text {th }}$ century witnesses a sharp rise in the incidence of mycobacterial infections, the development of new antimycobacterial drugs is presently of utmost importance and should proceed at a rapid pace. When exploring a possible link between antituberculous activity and the ability to form chelates with heavy metals, we prepared a set of N,N'-diarylethane- and -propanediamides, which were evaluated in vitro against Mycobacterium tuberculosis, and some of them showed significant activity [1]. Other authors have described various kinds of biological activity of alkanediamide derivatives as well. For example, some 2-methylcarbonylbutanediamides are active against $M$. tuberculosis [2], 2,3diarylpentanediamides display activity against Gram-positive bacteria [3], and N,N'-substituted 2halobutanediamides act as herbicides [4]. Raynes et al. studied the influence of the length of the connecting chain on the antimalarial activity of bisquinolines; the derivative of butanediamide was the most efficient [5]. In our previous study [6] we found that N,N'-bis(3,4-dichlorophenyl)butanediamide effectively inhibited oxygen evolution rate (OER) in spinach chloroplasts and that this compound interacted with the pigment-protein complexes in photosystem 2. The increase of the length of the connecting chain in the series of N,N'-bis(3,4-dichlorophenyl)alkanediamides led to the decrease of OERinhibiting activity in spinach chloroplast [7]. The decrease in biological activity with increasing lipophilicity of the compounds is probably linked to their lowered aqueous solubility, and hence to a restricted passage of the inhibitor through the hydrophilic regions of thylakoid membranes.

This study is focused on the synthesis of a large set of N,N'-diarylalkanediamides and on the study of antimycobacterial and antialgal activity of these compounds.

\section{Results and Discussion}

$\mathrm{N}, \mathrm{N}$--Diarylalkanediamides, with the exception of N,N'-diarylethanediamides, were prepared from the corresponding anilines by treatment with the appropriate alkanedioyl dichlorides in pyridine at $0^{\circ} \mathrm{C}$. The reaction mixtures were allowed to stand at room temperature and after 24 hours they were poured into water. The products were filtered off, washed with water and crystallized from ethanol. The alkanedioyl dichlorides were prepared from the corresponding acids by the reaction with phosphorus pentachloride (butanedioyl dichloride) or thionyl chloride (all other alkanedioyl dichlorides). N,N'Diarylethanediamides were prepared from diethyl oxalate by the reaction with the corresponding aniline in the presence of sodium ethanolate. All syntheses are outlined in Scheme 1. The yields, physical properties and analytical data for compounds 1-46 are given in Table 1 and Table 2. 


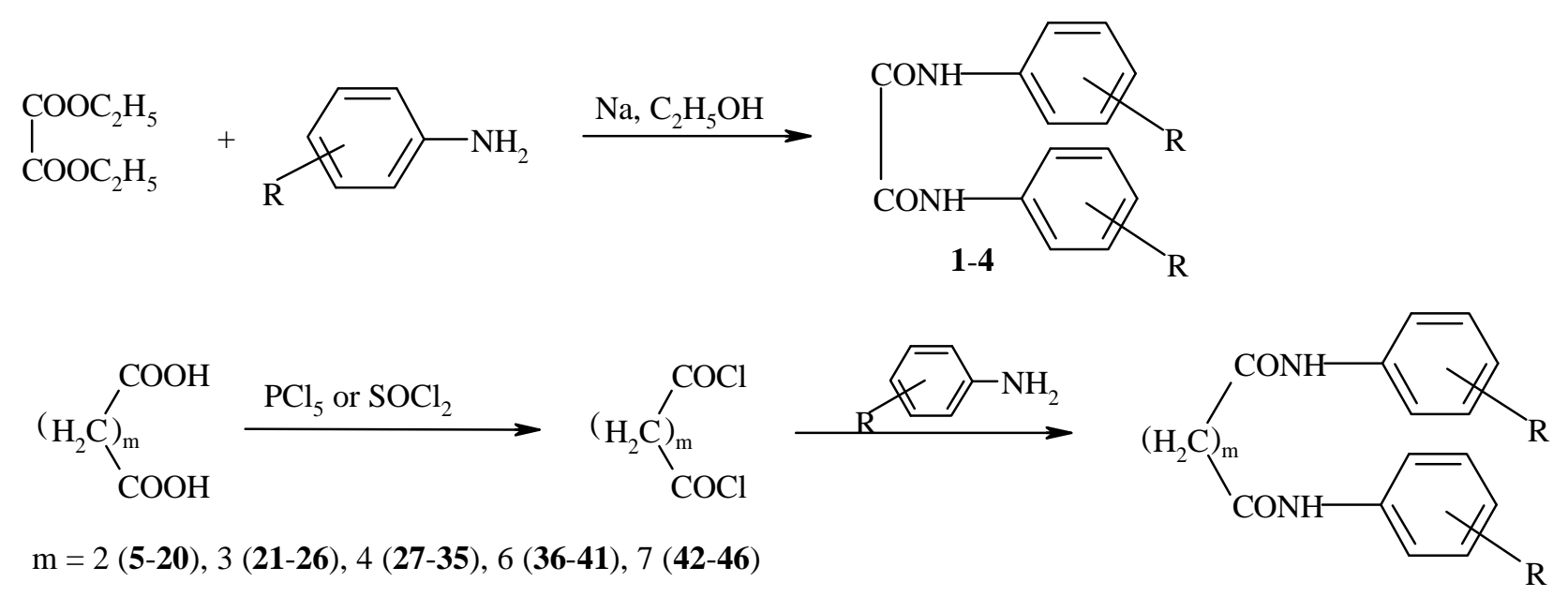

Scheme 1.

Compounds 1-46 were tested for their in vitro antimycobacterial activity against Mycobacterium tuberculosis, M. avium, M. fortuitum, and M. kansasii. We found that the antimycobacterial activity of $\mathrm{N}, \mathrm{N}$-diarylalkanediamides depends on the lipophilicity of the respective acid. Active substances were found only in the series of $\mathrm{N}, \mathrm{N}$-diarylethanediamides and $\mathrm{N}, \mathrm{N}$-diarylbutanediamides, but their antimycobacterial activity was mostly low. The MIC values of the active compounds are given in Table 3 . All derivatives of pentane-, hexane-, octane- and nonanediamide were inactive against the studied mycobacteria strains.

The inhibition of chlorophyll production in statically cultivated algae Chlorella vulgaris by selected

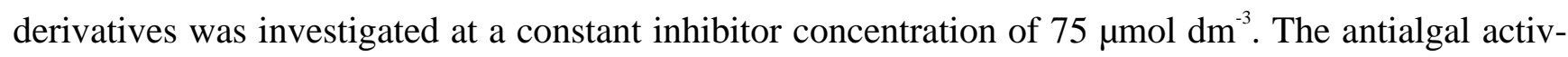
ity of the compounds was generally low, and the observed inhibition of algal chlorophyll production varied in the range of 14.2 (10) to 57.9\% (40) (Table 4 and Table 5). The antialgal activity of N,N'diarylbutanediamides was relatively low, varying in the range of 14.2 (10) to $43.6 \%$ (15) (Table 5). The most effective inhibitor from the series of N,N'-diarylalkanediamides was N,N'-bis(4methoxyphenyl)octanediamide (40), causing 57.9\% inhibition of chlorophyll production (Table 4). Antialgal activity of substituted N,N'-diarylalkanediamides with the same substituent $\mathrm{R}$ was proportional to the number of methylene groups in the connecting chain of the molecule $(\mathrm{m}=2-4,6,7)$ for derivatives with $\mathrm{R}=\mathrm{H}$ and 4-Cl, respectively. For derivatives with $\mathrm{R}=4-\mathrm{CH}_{3}, 4-\mathrm{OCH}_{3}$ and 3,4-Cl 2 , a quasi-parabolic dependence of the inhibitory activity on $\mathrm{m}$ was found, with maximum inhibition for $\mathrm{N}, \mathrm{N}$-diaryloctanediamides $\left(\mathrm{m}=6 ; \mathrm{R}=4-\mathrm{CH}_{3}\right.$ (39) and $4-\mathrm{OCH}_{3}$ (40)) and $\mathrm{N}, \mathrm{N}$ '-bis $(3,4-$ dichlorophenyl)hexanediamide $(\mathrm{m}=4 ; \mathbf{3 1})$. The relatively low biological activity of the compounds is probably a consequence of their low aqueous solubility, and hence a restricted passage of the inhibitor through the hydrophilic regions of thylakoid membranes. An efficient inhibition of photosynthetic electron transport in spinach chloroplasts by N,N'-bis(3,4-dichlorophenyl)butanediamide has been observed previously $[6,7]$. 
Table 1. Physical properties and analytical data of compounds 1-46.

\begin{tabular}{|c|c|c|c|c|c|c|c|}
\hline \multirow[t]{2}{*}{ Compd. } & \multirow[t]{2}{*}{$\begin{array}{l}\text { Formula } \\
\text { (M. w.) }\end{array}$} & \multirow[t]{2}{*}{$\mathrm{m}$} & \multirow[t]{2}{*}{$\mathrm{R}$} & \multirow[t]{2}{*}{$\begin{array}{l}\text { M.p. }\left({ }^{\circ} \mathrm{C}\right) \\
\text { (Yield, \%) }\end{array}$} & \multicolumn{3}{|c|}{$\begin{array}{l}\% \text { Calc. } \\
\% \text { Found }\end{array}$} \\
\hline & & & & & $\mathrm{C}$ & $\mathrm{H}$ & $\mathrm{N}$ \\
\hline $1^{\mathrm{a}}$ & $\begin{array}{c}\mathrm{C}_{16} \mathrm{H}_{16} \mathrm{~N}_{2} \mathrm{O}_{2} \\
(268.3)\end{array}$ & 0 & $2-\mathrm{CH}_{3}$ & $\begin{array}{c}211-212 \\
(65)\end{array}$ & - & - & - \\
\hline $2^{\mathrm{a}}$ & $\begin{array}{c}\mathrm{C}_{16} \mathrm{H}_{16} \mathrm{~N}_{2} \mathrm{O}_{2} \\
(268.3)\end{array}$ & 0 & $3-\mathrm{CH}_{3}$ & $\begin{array}{c}135-137 \\
(60)\end{array}$ & - & - & - \\
\hline $3^{\mathrm{a}}$ & $\begin{array}{c}\mathrm{C}_{14} \mathrm{H}_{10} \mathrm{~N}_{4} \mathrm{O}_{6} \\
(330.3)\end{array}$ & 0 & $4-\mathrm{NO}_{2}$ & $\begin{array}{c}357-358 \\
(61)\end{array}$ & - & - & - \\
\hline $4^{\mathrm{a}}$ & $\begin{array}{c}\mathrm{C}_{14} \mathrm{H}_{10} \mathrm{Br}_{2} \mathrm{~N}_{2} \mathrm{O}_{2} \\
(398.1)\end{array}$ & 0 & $4-\mathrm{Br}$ & $\begin{array}{c}329-331 \\
(58)\end{array}$ & - & - & - \\
\hline $5^{\mathrm{a}}$ & $\begin{array}{c}\mathrm{C}_{16} \mathrm{H}_{14} \mathrm{Br}_{2} \mathrm{~N}_{2} \mathrm{O}_{2} \\
(426.1)\end{array}$ & 2 & $4-\mathrm{Br}$ & $\begin{array}{c}281-282 \\
(92)\end{array}$ & - & - & - \\
\hline $6^{\mathrm{b}}$ & $\begin{array}{c}\mathrm{C}_{16} \mathrm{H}_{12} \mathrm{Cl}_{4} \mathrm{~N}_{2} \mathrm{O}_{2} \\
(406.1)\end{array}$ & 2 & $3,4-\mathrm{Cl}_{2}$ & $\begin{array}{c}258-259 \\
(93)\end{array}$ & - & - & - \\
\hline $7^{\mathrm{a}}$ & $\begin{array}{c}\mathrm{C}_{16} \mathrm{H}_{16} \mathrm{~N}_{2} \mathrm{O}_{2} \\
(268.3)\end{array}$ & 2 & $\mathrm{H}$ & $\begin{array}{c}231-233 \\
(95)\end{array}$ & - & - & - \\
\hline $8^{\mathrm{a}}$ & $\begin{array}{c}\mathrm{C}_{18} \mathrm{H}_{20} \mathrm{~N}_{2} \mathrm{O}_{4} \\
(328.4)\end{array}$ & 2 & $4-\mathrm{OCH}_{3}$ & $\begin{array}{c}256-257 \\
(90)\end{array}$ & - & - & - \\
\hline 9 & $\begin{array}{c}\mathrm{C}_{16} \mathrm{H}_{14} \mathrm{Cl}_{2} \mathrm{~N}_{2} \mathrm{O}_{2} \\
(337.2)\end{array}$ & 2 & $4-\mathrm{Cl}$ & $\begin{array}{c}288-290 \\
(90)\end{array}$ & $\begin{array}{l}56.99 \\
57.24\end{array}$ & $\begin{array}{l}4.18 \\
4.15\end{array}$ & $\begin{array}{l}8.31 \\
8.38\end{array}$ \\
\hline 10 & $\begin{array}{c}\mathrm{C}_{18} \mathrm{H}_{20} \mathrm{~N}_{2} \mathrm{O}_{2} \\
(296.4)\end{array}$ & 2 & $4-\mathrm{CH}_{3}$ & $\begin{array}{c}273-275 \\
(91)\end{array}$ & $\begin{array}{l}72.95 \\
72.87\end{array}$ & $\begin{array}{l}6.80 \\
6.79\end{array}$ & $\begin{array}{l}9.45 \\
9.59\end{array}$ \\
\hline $11^{\mathrm{b}}$ & $\begin{array}{c}\mathrm{C}_{16} \mathrm{H}_{14} \mathrm{~F}_{2} \mathrm{~N}_{2} \mathrm{O}_{2} \\
(304.3)\end{array}$ & 2 & $3-\mathrm{F}$ & $\begin{array}{c}206-207 \\
(89)\end{array}$ & - & - & - \\
\hline $12^{\mathrm{b}}$ & $\begin{array}{c}\mathrm{C}_{16} \mathrm{H}_{14} \mathrm{~F}_{2} \mathrm{~N}_{2} \mathrm{O}_{2} \\
(304.3)\end{array}$ & 2 & $4-\mathrm{F}$ & $\begin{array}{c}244-245 \\
(92)\end{array}$ & - & - & - \\
\hline $13^{\mathrm{b}}$ & $\begin{array}{c}\mathrm{C}_{16} \mathrm{H}_{14} \mathrm{~N}_{4} \mathrm{O}_{6} \\
(358.3)\end{array}$ & 2 & $3-\mathrm{NO}_{2}$ & $\begin{array}{c}228-230 \\
(88)\end{array}$ & - & - & - \\
\hline $14^{\mathrm{b}}$ & $\begin{array}{c}\mathrm{C}_{20} \mathrm{H}_{24} \mathrm{~N}_{2} \mathrm{O}_{2} \\
(324.4)\end{array}$ & 2 & $3,4-\left(\mathrm{CH}_{3}\right)_{2}$ & $\begin{array}{c}231-232 \\
(85)\end{array}$ & - & - & - \\
\hline $15^{\mathrm{a}}$ & $\begin{array}{c}\mathrm{C}_{16} \mathrm{H}_{14} \mathrm{Cl}_{2} \mathrm{~N}_{2} \mathrm{O}_{2} \\
(337.2)\end{array}$ & 2 & $3-\mathrm{Cl}$ & $\begin{array}{c}233-235 \\
(92)\end{array}$ & - & - & - \\
\hline $16^{\mathrm{b}}$ & $\begin{array}{c}\mathrm{C}_{24} \mathrm{H}_{32} \mathrm{~N}_{2} \mathrm{O}_{2} \\
(380.5)\end{array}$ & 2 & $4-\mathrm{C}_{4} \mathrm{H}_{9}$ & $\begin{array}{c}232-234 \\
(81)\end{array}$ & - & - & - \\
\hline
\end{tabular}


Continuation of the Table 1.

\begin{tabular}{|c|c|c|c|c|c|c|c|}
\hline \multirow[t]{2}{*}{ Compd. } & \multirow[t]{2}{*}{$\begin{array}{c}\text { Formula } \\
\text { (M. w.) }\end{array}$} & \multirow[t]{2}{*}{$\mathrm{m}$} & \multirow[t]{2}{*}{$\mathrm{R}$} & \multirow[t]{2}{*}{$\begin{array}{l}\text { M.p. }\left({ }^{\circ} \mathrm{C}\right) \\
\text { (Yield, \%) }\end{array}$} & \multicolumn{3}{|c|}{$\begin{array}{l}\% \text { Calc. } \\
\% \text { Found }\end{array}$} \\
\hline & & & & & $\mathrm{C}$ & $\mathrm{H}$ & $\mathrm{N}$ \\
\hline $17^{\mathrm{b}}$ & $\begin{array}{c}\mathrm{C}_{22} \mathrm{H}_{28} \mathrm{~N}_{2} \mathrm{O}_{2} \\
(352.5)\end{array}$ & 2 & 4-isoC ${ }_{3} \mathrm{H}_{7}$ & $\begin{array}{c}234-236 \\
(83)\end{array}$ & - & - & - \\
\hline $18^{\mathrm{b}}$ & $\begin{array}{c}\mathrm{C}_{24} \mathrm{H}_{32} \mathrm{~N}_{2} \mathrm{O}_{2} \\
(380.5)\end{array}$ & 2 & $4-\mathrm{sec}-\mathrm{C}_{4} \mathrm{H}_{9}$ & $\begin{array}{c}178-179 \\
(81)\end{array}$ & - & - & - \\
\hline $19^{\mathrm{a}}$ & $\begin{array}{c}\mathrm{C}_{20} \mathrm{H}_{26} \mathrm{~N}_{4} \mathrm{O}_{2} \\
(354.5)\end{array}$ & 2 & $4-\mathrm{N}\left(\mathrm{CH}_{3}\right)_{2}$ & $\begin{array}{c}282.5-283.5 \\
(88)\end{array}$ & - & - & - \\
\hline 20 & $\begin{array}{c}\mathrm{C}_{18} \mathrm{H}_{14} \mathrm{~N}_{4} \mathrm{O}_{2} \mathrm{~S}_{2} \\
(382.5)\end{array}$ & 2 & $\mathrm{c}$ & $\begin{array}{c}282-285 \\
(92)\end{array}$ & $\begin{array}{l}56.53 \\
56.55\end{array}$ & $\begin{array}{l}3.69 \\
3.87\end{array}$ & $\begin{array}{l}14.65 \\
14.88\end{array}$ \\
\hline 21 & $\begin{array}{c}\mathrm{C}_{17} \mathrm{H}_{16} \mathrm{Cl}_{2} \mathrm{~N}_{2} \mathrm{O}_{2} \\
(351.2)\end{array}$ & 3 & $4-\mathrm{Cl}$ & $\begin{array}{c}242-243 \\
(70)\end{array}$ & $\begin{array}{l}58.13 \\
58.13\end{array}$ & $\begin{array}{l}4.59 \\
4.59\end{array}$ & $\begin{array}{l}7.98 \\
7.98\end{array}$ \\
\hline 22 & $\begin{array}{c}\mathrm{C}_{19} \mathrm{H}_{22} \mathrm{~N}_{2} \mathrm{O}_{4} \\
(342.4)\end{array}$ & 3 & $4-\mathrm{OCH}_{3}$ & $\begin{array}{c}222.5-224 \\
(62)\end{array}$ & $\begin{array}{l}66.65 \\
66.93\end{array}$ & $\begin{array}{l}6.48 \\
6.45\end{array}$ & $\begin{array}{l}8.18 \\
8.24\end{array}$ \\
\hline $23^{\mathrm{a}}$ & $\begin{array}{c}\mathrm{C}_{17} \mathrm{H}_{18} \mathrm{~N}_{2} \mathrm{O}_{2} \\
(282.3)\end{array}$ & 3 & $\mathrm{H}$ & $\begin{array}{c}224-225 \\
(84)\end{array}$ & - & - & - \\
\hline $24^{\mathrm{a}}$ & $\begin{array}{c}\mathrm{C}_{19} \mathrm{H}_{22} \mathrm{~N}_{2} \mathrm{O}_{2} \\
(310.4)\end{array}$ & 3 & $4-\mathrm{CH}_{3}$ & $\begin{array}{c}220-2211 \\
(54)\end{array}$ & - & - & - \\
\hline $25^{\mathrm{b}}$ & $\begin{array}{c}\mathrm{C}_{17} \mathrm{H}_{14} \mathrm{Cl}_{4} \mathrm{~N}_{2} \mathrm{O}_{2} \\
(420.1)\end{array}$ & 3 & $3,4-\mathrm{Cl}_{2}$ & $\begin{array}{c}266-267 \\
(63)\end{array}$ & - & - & - \\
\hline $26^{\mathrm{a}}$ & $\begin{array}{c}\mathrm{C}_{17} \mathrm{H}_{16} \mathrm{Br}_{2} \mathrm{~N}_{2} \mathrm{O}_{2} \\
(440.1)\end{array}$ & 3 & $4-\mathrm{Br}$ & $\begin{array}{c}256-257 \\
(58)\end{array}$ & - & - & - \\
\hline 27 & $\begin{array}{c}\mathrm{C}_{20} \mathrm{H}_{24} \mathrm{~N}_{2} \mathrm{O}_{2} \\
(324.4)\end{array}$ & 4 & $4-\mathrm{CH}_{3}$ & $\begin{array}{c}258-259 \\
(67)\end{array}$ & $\begin{array}{l}74.05 \\
74.21\end{array}$ & $\begin{array}{l}7.46 \\
7.52\end{array}$ & $\begin{array}{l}8.63 \\
8.66\end{array}$ \\
\hline $28^{\mathrm{a}}$ & $\begin{array}{c}\mathrm{C}_{18} \mathrm{H}_{20} \mathrm{~N}_{2} \mathrm{O}_{2} \\
(296.4)\end{array}$ & 4 & $\mathrm{H}$ & $\begin{array}{c}237-238 \\
(75)\end{array}$ & - & - & - \\
\hline 29 & $\begin{array}{c}\mathrm{C}_{18} \mathrm{H}_{18} \mathrm{~N}_{4} \mathrm{O}_{6} \\
(386.4)\end{array}$ & 4 & $3-\mathrm{NO}_{2}$ & $\begin{array}{c}238-239 \\
(58)\end{array}$ & $\begin{array}{l}55.96 \\
55.80\end{array}$ & $\begin{array}{l}4.70 \\
4.73\end{array}$ & $\begin{array}{l}14.50 \\
14.67\end{array}$ \\
\hline 30 & $\begin{array}{c}\mathrm{C}_{18} \mathrm{H}_{18} \mathrm{Cl}_{2} \mathrm{~N}_{2} \mathrm{O}_{2} \\
(365.3)\end{array}$ & 4 & $3-\mathrm{Cl}$ & $\begin{array}{c}197-198 \\
(60)\end{array}$ & $\begin{array}{l}59.19 \\
59.14\end{array}$ & $\begin{array}{l}4.97 \\
4.97\end{array}$ & $\begin{array}{l}7.67 \\
7.90\end{array}$ \\
\hline $31^{\mathrm{b}}$ & $\begin{array}{c}\mathrm{C}_{18} \mathrm{H}_{16} \mathrm{Cl}_{4} \mathrm{~N}_{2} \mathrm{O}_{2} \\
(434.2)\end{array}$ & 4 & $3,4-\mathrm{Cl}_{2}$ & $\begin{array}{c}276-277 \\
(59)\end{array}$ & - & - & - \\
\hline 32 & $\begin{array}{c}\mathrm{C}_{20} \mathrm{H}_{24} \mathrm{~N}_{2} \mathrm{O}_{4} \\
(356.4)\end{array}$ & 4 & $2-\mathrm{OCH}_{3}$ & $\begin{array}{c}151-152 \\
(40)\end{array}$ & $\begin{array}{l}67.40 \\
67.49\end{array}$ & $\begin{array}{l}6.79 \\
6.68 \\
\end{array}$ & $\begin{array}{l}7.86 \\
7.79 \\
\end{array}$ \\
\hline
\end{tabular}


Continuation of the Table 1.

\begin{tabular}{|c|c|c|c|c|c|c|c|}
\hline \multirow[t]{2}{*}{ Compd. } & \multirow[t]{2}{*}{$\begin{array}{l}\text { Formula } \\
\text { (M. w.) }\end{array}$} & \multirow[t]{2}{*}{$\mathrm{m}$} & \multirow[t]{2}{*}{$\mathrm{R}$} & \multirow[t]{2}{*}{$\begin{array}{l}\text { M.p. }\left({ }^{\circ} \mathrm{C}\right) \\
\text { (Yield, \%) }\end{array}$} & \multicolumn{3}{|c|}{$\begin{array}{l}\% \text { Calc. } \\
\% \text { Found }\end{array}$} \\
\hline & & & & & $\mathrm{C}$ & $\mathrm{H}$ & $\mathrm{N}$ \\
\hline \multirow[t]{2}{*}{33} & $\mathrm{C}_{20} \mathrm{H}_{24} \mathrm{~N}_{2} \mathrm{O}_{4}$ & 4 & $4-\mathrm{OCH}_{3}$ & $233-234$ & 67.40 & 6.79 & 7.86 \\
\hline & $(356.4)$ & & & $(65)$ & 67.62 & 6.78 & 7.88 \\
\hline \multirow[t]{2}{*}{34} & $\mathrm{C}_{18} \mathrm{H}_{18} \mathrm{Cl}_{2} \mathrm{~N}_{2} \mathrm{O}_{2}$ & 4 & $4-\mathrm{Cl}$ & $238-239$ & 59.19 & 4.97 & 7.67 \\
\hline & (356.3) & & & (67) & 58.91 & 4.82 & 7.79 \\
\hline \multirow[t]{2}{*}{35} & $\mathrm{C}_{24} \mathrm{H}_{28} \mathrm{~N}_{2} \mathrm{O}_{6}$ & 4 & $4-$ & $216-217$ & 65.44 & 6.41 & 6.36 \\
\hline & $(440.5)$ & & $\mathrm{COOC}_{2} \mathrm{H}_{5}$ & $(54)$ & 65.70 & 6.33 & 6.17 \\
\hline $36^{\mathrm{b}}$ & $\begin{array}{c}\mathrm{C}_{20} \mathrm{H}_{20} \mathrm{Cl}_{4} \mathrm{~N}_{2} \mathrm{O}_{2} \\
(462.2)\end{array}$ & 6 & $3,4-\mathrm{Cl}_{2}$ & $\begin{array}{c}167-168 \\
(35)\end{array}$ & - & - & - \\
\hline \multirow[t]{2}{*}{37} & $\mathrm{C}_{20} \mathrm{H}_{22} \mathrm{Cl}_{2} \mathrm{~N}_{2} \mathrm{O}_{2}$ & 6 & $4-\mathrm{Cl}$ & $197-198$ & 61.11 & 5.60 & 7.12 \\
\hline & (393.3) & & & (81) & 61.08 & 5.64 & 7.12 \\
\hline $38^{\mathrm{a}}$ & $\begin{array}{c}\mathrm{C}_{20} \mathrm{H}_{24} \mathrm{~N}_{2} \mathrm{O}_{2} \\
(324.4)\end{array}$ & 6 & $\mathrm{H}$ & $\begin{array}{c}186-188 \\
(57)\end{array}$ & - & - & - \\
\hline $39^{\mathrm{a}}$ & $\begin{array}{c}\mathrm{C}_{22} \mathrm{H}_{28} \mathrm{~N}_{2} \mathrm{O}_{2} \\
(352.5)\end{array}$ & 6 & $4-\mathrm{CH}_{3}$ & $\begin{array}{c}224-226 \\
(55)\end{array}$ & - & - & - \\
\hline \multirow[t]{2}{*}{40} & $\mathrm{C}_{22} \mathrm{H}_{28} \mathrm{~N}_{2} \mathrm{O}_{4}$ & 6 & $4-\mathrm{OCH}_{3}$ & $220-221$ & 68.78 & 7.24 & 7.29 \\
\hline & (384.5) & & & (63) & 68.60 & 7.15 & 7.19 \\
\hline $41^{\mathrm{a}}$ & $\begin{array}{c}\mathrm{C}_{20} \mathrm{H}_{22} \mathrm{Br}_{2} \mathrm{~N}_{2} \mathrm{O}_{2} \\
(482.2)\end{array}$ & 6 & $4-\mathrm{Br}$ & $\begin{array}{c}251-253 \\
(54)\end{array}$ & - & - & - \\
\hline $42^{\mathrm{b}}$ & $\begin{array}{c}\mathrm{C}_{21} \mathrm{H}_{22} \mathrm{Cl}_{4} \mathrm{~N}_{2} \mathrm{O}_{2} \\
(476.2)\end{array}$ & 7 & $3,4-\mathrm{Cl}_{2}$ & $\begin{array}{c}170-171 \\
(52)\end{array}$ & - & - & - \\
\hline \multirow[t]{2}{*}{43} & $\mathrm{C}_{21} \mathrm{H}_{24} \mathrm{Cl}_{2} \mathrm{~N}_{2} \mathrm{O}_{2}$ & 7 & $4-\mathrm{Cl}$ & 196-197 & 61.92 & 5.94 & 6.88 \\
\hline & $(407.3)$ & & & $(61)$ & 62.06 & 5.85 & 6.99 \\
\hline $44^{\mathrm{a}}$ & $\begin{array}{c}\mathrm{C}_{21} \mathrm{H}_{26} \mathrm{~N}_{2} \mathrm{O}_{2} \\
(338.5)\end{array}$ & 7 & $\mathrm{H}$ & $\begin{array}{c}180-181 \\
(63)\end{array}$ & - & - & - \\
\hline $45^{\mathrm{a}}$ & $\begin{array}{c}\mathrm{C}_{23} \mathrm{H}_{30} \mathrm{~N}_{2} \mathrm{O}_{2} \\
\quad(366.5)\end{array}$ & 7 & $4-\mathrm{CH}_{3}$ & $\begin{array}{c}197-198 \\
(61)\end{array}$ & - & - & - \\
\hline \multirow[t]{2}{*}{46} & $\mathrm{C}_{23} \mathrm{H}_{30} \mathrm{~N}_{2} \mathrm{O}_{4}$ & 7 & $4-\mathrm{OCH}_{3}$ & 194-196 & 69.32 & 7.59 & 7.03 \\
\hline & $(398.5)$ & & & $(44)$ & 69.61 & 7.73 & 7.07 \\
\hline
\end{tabular}

${ }^{\mathrm{a}}$ M. p. values from literature: Compound, value ( $\left.{ }^{\circ} \mathrm{C}\right)$ [ref.]: 1, 209 [8]; 2, 133 [8]; 3, 359 [9]; 4, 321-322 [10]; 5, 284 [11]; 7, 230,5 [12]; 8, 256 [13]; 15, 232 [14]; 19, 277-280 [15]; 23, 223 [11]; 24, 218 [11]; 26, 256 [11]; 28, 235 [11]; 38, 186-7 [16]; 39, 219 [11]; 41, 248 [11]; 44, 186-7 [16]; 45, 198 [11]. ${ }^{\mathrm{b}}$ The data of the compound were taken from [7].

'The compound is N,N'-bis(2-benzothiazolyl)butanediamide. 
Table 2. ${ }^{1} \mathrm{H}$ NMR and IR spectroscopic data.

\begin{tabular}{|c|c|c|}
\hline Compd. & $\begin{array}{l}{ }^{1} \mathrm{H} \text { NMR } \\
\delta(\mathrm{ppm})\end{array}$ & $\begin{array}{c}\mathrm{IR} \\
\mathrm{v}(\mathrm{C}=\mathrm{O})\left(\mathrm{cm}^{-1}\right)\end{array}$ \\
\hline 1 & - & 1668 \\
\hline 2 & - & 1666 \\
\hline 3 & - & 1706 \\
\hline 4 & - & 1666 \\
\hline 5 & - & 1652 \\
\hline 6 & - & 1659 \\
\hline 7 & - & 1663 \\
\hline 8 & - & 1648 \\
\hline 9 & $\begin{array}{c}2.63(\mathrm{~s}, 4 \mathrm{H}), 7.35-7.28(\mathrm{~m}, 4 \mathrm{H}), 7.63-7.56(\mathrm{~m}, 4 \mathrm{H}), 10.14(\mathrm{~s} \\
2 \mathrm{H})\end{array}$ & 1652 \\
\hline 10 & $\begin{array}{c}2.21(\mathrm{~s}, 6 \mathrm{H}), 2.60(\mathrm{~s}, 4 \mathrm{H}), 7.11-7.03(\mathrm{~m}, 4 \mathrm{H}), 7.48-7.40(\mathrm{~m}, \\
4 \mathrm{H}), 9.89(\mathrm{~s}, 2 \mathrm{H})\end{array}$ & 1655 \\
\hline 11 & - & 1663 \\
\hline 12 & - & 1651 \\
\hline 13 & - & 1675 \\
\hline 14 & - & 1651 \\
\hline 15 & - & 1668 \\
\hline 16 & - & 1659 \\
\hline 17 & - & 1656 \\
\hline 18 & - & 1655 \\
\hline 19 & - & 1645 \\
\hline 20 & $\begin{array}{c}2.88(\mathrm{~s}, 4 \mathrm{H}), 7.32-7.23(\mathrm{~m}, 2 \mathrm{H}), 7.46-7.37(\mathrm{~m}, 2 \mathrm{H}), 7.76-7.69 \\
(\mathrm{~m}, 2 \mathrm{H}), 7.97-7.90(\mathrm{~m}, 2 \mathrm{H})\end{array}$ & 1694 \\
\hline 21 & $\begin{array}{c}1.95-1.81(\mathrm{~m}, 2 \mathrm{H}), 2.36(\mathrm{t}, \mathrm{J}=7.4 \mathrm{~Hz}, 4 \mathrm{H}), 7.35-7.29(\mathrm{~m}, 4 \mathrm{H}) \\
7.64-7.57(\mathrm{~m}, 4 \mathrm{H}), 10.05(\mathrm{~s}, 2 \mathrm{H})\end{array}$ & 1664 \\
\hline 22 & $\begin{array}{c}1.93-1.81(\mathrm{~m}, 2 \mathrm{H}), 2.31(\mathrm{t}, \mathrm{J}=7.4 \mathrm{~Hz}, 4 \mathrm{H}), 3.69(\mathrm{~s}, 6 \mathrm{H}), 6.89- \\
6.79(\mathrm{~m}, 4 \mathrm{H}), 7.53-7.43(\mathrm{~m}, 4 \mathrm{H}), 9.76(\mathrm{~s}, 2 \mathrm{H})\end{array}$ & 1659 \\
\hline 23 & - & 1673 \\
\hline 24 & - & 1664 \\
\hline 25 & - & 1679 \\
\hline 26 & 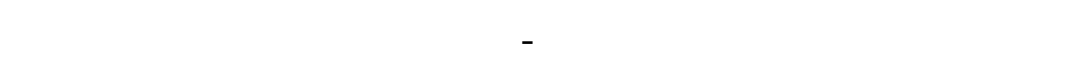 & 1664 \\
\hline 27 & $\begin{array}{c}1.63-1.55(\mathrm{~m}, 4 \mathrm{H}), 2.21(\mathrm{~s}, 6 \mathrm{H}), 2.33-2.24(\mathrm{~m}, 4 \mathrm{H}), 7.10-7.03 \\
(\mathrm{~m}, 4 \mathrm{H}), 7.48-7.41(\mathrm{~m}, 4 \mathrm{H}), 9.79(\mathrm{~s}, 2 \mathrm{H})\end{array}$ & 1659 \\
\hline 28 & - & 1660 \\
\hline
\end{tabular}


Continuation of the Table 2.

\begin{tabular}{|c|c|c|}
\hline Compd. & $\begin{array}{l}{ }^{1} \mathrm{H} \text { NMR } \\
\delta(\mathrm{ppm})\end{array}$ & $\begin{array}{c}\mathrm{IR} \\
\mathrm{v}(\mathrm{C}=\mathrm{O})\left(\mathrm{cm}^{-1}\right)\end{array}$ \\
\hline 29 & $\begin{array}{c}1.70-1.60(\mathrm{~m}, 4 \mathrm{H}), 2.43-2.33(\mathrm{~m}, 4 \mathrm{H}), 7.57(\mathrm{t}, \mathrm{J}=8.1,2 \mathrm{H}), \\
7.91-7.83(\mathrm{~m}, 4 \mathrm{H}), 8.63(\mathrm{t}, \mathrm{J}=2.1 \mathrm{~Hz}, 2 \mathrm{H}), 10.41(\mathrm{~s}, 2 \mathrm{H})\end{array}$ & 1667 \\
\hline 30 & $\begin{array}{c}1.65-1.55(\mathrm{~m}, 4 \mathrm{H}), 2.38-2.27(\mathrm{~m}, 4 \mathrm{H}), 7.10-7.03(\mathrm{~m}, 2 \mathrm{H}), 7.30 \\
(\mathrm{t}, \mathrm{J}=8.1,2 \mathrm{H}), 7.45-7.38(\mathrm{~m}, 2 \mathrm{H}), 7.80(\mathrm{t}, \mathrm{J}=1.9,2 \mathrm{H}), 10.09(\mathrm{~s} \\
2 \mathrm{H})\end{array}$ & 1663 \\
\hline 31 & - & 1670 \\
\hline 32 & $\begin{array}{c}1.65-1.54(\mathrm{~m}, 4 \mathrm{H}), 2.44-2.33(\mathrm{~m}, 4 \mathrm{H}), 3.79(\mathrm{~s}, 6 \mathrm{H}), 6.93-6.82 \\
(\mathrm{~m}, 2 \mathrm{H}), 7.10-6.96(\mathrm{~m}, 4 \mathrm{H}), 7.97-7.84(\mathrm{~m}, 2 \mathrm{H}), 9.03(\mathrm{~s}, 2 \mathrm{H})\end{array}$ & 1659 \\
\hline 33 & $\begin{array}{c}1.64-1.52(\mathrm{~m}, 4 \mathrm{H}), 2.33-2.20(\mathrm{~m}, 4 \mathrm{H}), 3.69(\mathrm{~s}, 6 \mathrm{H}), 6.89-6.79 \\
(\mathrm{~m}, 4 \mathrm{H}), 7.52-7.42(\mathrm{~m}, 4 \mathrm{H}), 9.74(\mathrm{~s}, 2 \mathrm{H})\end{array}$ & 1648 \\
\hline 34 & $\begin{array}{c}1.65-1.55(\mathrm{~m}, 4 \mathrm{H}), 2.37-2.26(\mathrm{~m}, 4 \mathrm{H}), 7.35-7.29(\mathrm{~m}, 4 \mathrm{H}), 7.63- \\
7.56(\mathrm{~m}, 4 \mathrm{H}), 10.03(\mathrm{~s}, 2 \mathrm{H})\end{array}$ & 1656 \\
\hline 35 & $\begin{array}{c}1.28(\mathrm{t}, \mathrm{J}=7.1 \mathrm{~Hz}, 6 \mathrm{H}), 1.68-1.57(\mathrm{~m}, 4 \mathrm{H}), 2.42-2.31(\mathrm{~m}, 4 \mathrm{H}) \\
4.25(\mathrm{q}, \mathrm{J}=14.2, \mathrm{~J}=7.1 \mathrm{~Hz}, 4 \mathrm{H}), 7.74-7.67(\mathrm{~m}, 4 \mathrm{H}), 7.91-7.84 \\
(\mathrm{~m}, 4 \mathrm{H}), 10.25(\mathrm{~s}, 2 \mathrm{H})\end{array}$ & 1708,1693 \\
\hline 36 & - & 1671 \\
\hline 37 & $\begin{array}{c}1.36-1.25(\mathrm{~m}, 4 \mathrm{H}), 1.64-1.49(\mathrm{~m}, 4 \mathrm{H}), 2.27(\mathrm{t}, \mathrm{J}=7.4,4 \mathrm{H}), \\
7.35-7.27(\mathrm{~m}, 4 \mathrm{H}), 7.63-7.55(\mathrm{~m}, 4 \mathrm{H}), 10.00(\mathrm{~s}, 2 \mathrm{H})\end{array}$ & 1659 \\
\hline 38 & - & 1659 \\
\hline 39 & - & 1656 \\
\hline 40 & $\begin{array}{c}1.34-1.24(\mathrm{~m}, 4 \mathrm{H}), 1.64-1.48(\mathrm{~m}, 4 \mathrm{H}), 2.24(\mathrm{t}, \mathrm{J}=7.4,4 \mathrm{H}), 3.68 \\
(\mathrm{~s}, 6 \mathrm{H}), 6.87-6.79(\mathrm{~m}, 4 \mathrm{H}), 7.50-7.42(\mathrm{~m}, 4 \mathrm{H}), 9.72(\mathrm{~s}, 2 \mathrm{H})\end{array}$ & 1652 \\
\hline 41 & - & 1664 \\
\hline 42 & - & 1680 \\
\hline 43 & $\begin{array}{c}1.32-1.22(\mathrm{~m}, 6 \mathrm{H}), 1.63-1.48(\mathrm{~m}, 4 \mathrm{H}), 2.27(\mathrm{t}, \mathrm{J}=7.3 \mathrm{~Hz}, 4 \mathrm{H}), \\
7.34-7.28(\mathrm{~m}, 4 \mathrm{H}), 7.62-7.55(\mathrm{~m}, 4 \mathrm{H}), 10.00(\mathrm{~s}, 2 \mathrm{H})\end{array}$ & 1660 \\
\hline 44 & - & 1671 \\
\hline 45 & - & 1662 \\
\hline 46 & $\begin{array}{c}1.35-1.20(\mathrm{~m}, 6 \mathrm{H}), 1.63-1.47(\mathrm{~m}, 4 \mathrm{H}), 2.23(\mathrm{t}, \mathrm{J}=7.3 \mathrm{~Hz}, 4 \mathrm{H}) \\
3.68(\mathrm{~s}, 6 \mathrm{H}), 6.88-6.80(\mathrm{~m}, 4 \mathrm{H}), 7.50-7.42(\mathrm{~m}, 4 \mathrm{H}), 9.71(\mathrm{~s} \\
2 \mathrm{H})\end{array}$ & 1655 \\
\hline
\end{tabular}


Table 3. Antimycobacterial activity of the active compounds expressed as MIC $\left(\mu \mathrm{mol} \mathrm{dm} \mathrm{dm}^{-3}\right)$. ( $\mathrm{m}=$ number of methylene groups in the connecting chain of the compounds).

\begin{tabular}{|c|c|c|c|c|c|c|}
\hline \multirow[t]{2}{*}{ Compound } & \multirow[t]{2}{*}{$\mathrm{m}$} & \multirow[t]{2}{*}{$\mathrm{R}$} & \multicolumn{4}{|c|}{$\left.\mathrm{MIC}(\mu \mathrm{mol} \mathrm{dm})^{-3}\right)$} \\
\hline & & & M. tuberculosis & M. avium & M. kansasii & M. fortuitum \\
\hline 3 & 0 & $4-\mathrm{NO}_{2}$ & 37 & a) & -a) & $-^{\text {a) }}$ \\
\hline 4 & 0 & $4-\mathrm{Br}$ & 4.1 & $-{ }^{\text {a) }}$ & $-{ }^{\text {a) }}$ & $-{ }^{a)}$ \\
\hline 6 & 2 & $3,4-\mathrm{Cl}_{2}$ & 250 & 250 & 500 & 500 \\
\hline 7 & 2 & $\mathrm{H}$ & 500 & 500 & 500 & 500 \\
\hline 12 & 2 & $4-\mathrm{F}$ & 250 & 1000 & 1000 & 1000 \\
\hline 14 & 2 & $3,4-\left(\mathrm{CH}_{3}\right)_{2}$ & 500 & $>1000$ & $>1000$ & $>1000$ \\
\hline 15 & 2 & $3-\mathrm{Cl}$ & 500 & $>1000$ & $>1000$ & $>1000$ \\
\hline 16 & 2 & $4-\mathrm{C}_{4} \mathrm{H}_{9}$ & 500 & 63 & $>1000$ & $>1000$ \\
\hline 17 & 2 & 4-isoC ${ }_{3} \mathrm{H}_{7}$ & $>1000$ & 63 & $>1000$ & $>1000$ \\
\hline 18 & 2 & 4-sec- $\mathrm{C}_{4} \mathrm{H}_{9}$ & 500 & 63 & $>1000$ & $>1000$ \\
\hline
\end{tabular}

a) not tested

Table 4. Inhibition of chlorophyll production in Chlorella vulgaris by N,N'-diarylalkanediamides $(\mathrm{m}=$ number of methylene groups in the connecting chain of the compounds; concentrations of compounds were constant, $75 \mu \mathrm{mol} \mathrm{dm}^{-3}$ ).

\begin{tabular}{cccccc}
\hline \multirow{2}{*}{$\mathrm{m}$} & \multicolumn{5}{c}{$\begin{array}{c}\text { Compound } \\
\text { \% of inhibition }\end{array}$} \\
\cline { 2 - 6 } & $\mathrm{R}=\mathrm{H}$ & $4-\mathrm{CH}_{3}$ & $4-\mathrm{OCH}_{3}$ & $4-\mathrm{Cl}$ & $3,4-\mathrm{Cl}_{2}$ \\
\hline \multirow{2}{*}{2} & $\mathbf{7}$ & $\mathbf{1 0}$ & $\mathbf{8}$ & $\mathbf{9}$ & $\mathbf{6}$ \\
& 22.8 & 14.2 & 30.8 & 33.2 & 19.8 \\
3 & $\mathbf{2 3}$ & $\mathbf{2 4}$ & $\mathbf{2 2}$ & $\mathbf{2 1}$ & $\mathbf{2 5}$ \\
& 41.8 & 26.2 & 41.6 & 34.3 & 28.7 \\
4 & $\mathbf{2 8}$ & $\mathbf{2 7}$ & $\mathbf{3 3}$ & $\mathbf{3 4}$ & $\mathbf{3 1}$ \\
& 41.0 & 37.7 & 46.0 & 41.4 & 33.5 \\
6 & $\mathbf{3 8}$ & $\mathbf{3 9}$ & $\mathbf{4 0}$ & $\mathbf{3 7}$ & $\mathbf{3 6}$ \\
& 44.2 & 41.4 & 57.9 & 45.4 & 22.6 \\
& $\mathbf{4 4}$ & $\mathbf{4 5}$ & $\mathbf{4 6}$ & $\mathbf{4 3}$ & $\mathbf{4 2}$ \\
& 48.5 & 37.9 & 53.9 & 45.5 & 15.1 \\
\hline
\end{tabular}


Table 5. Inhibition of chlorophyll production in Chlorella vulgaris by $\mathrm{N}, \mathrm{N}$-diarylbutanediamides $\left(\mathrm{m}=2\right.$; concentrations of compounds were constant, $\left.75 \mu \mathrm{mol} \mathrm{dm}{ }^{-3}\right)$.

\begin{tabular}{cccccc}
\hline Compound & $\mathrm{R}$ & \% of inhibition & Compound & $\mathrm{R}$ & \% of inhibition \\
\hline $\mathbf{7}$ & $\mathrm{H}$ & 22.8 & $\mathbf{5}$ & $4-\mathrm{Br}$ & 27.1 \\
$\mathbf{1 1}$ & $3-\mathrm{F}$ & 25.2 & $\mathbf{1 7}$ & $4-\mathrm{isoC}_{3} \mathrm{H}_{7}$ & 36.5 \\
$\mathbf{1 5}$ & $3-\mathrm{Cl}$ & 43.6 & $\mathbf{1 6}$ & $4-\mathrm{C}_{4} \mathrm{H}_{9}$ & 32.6 \\
$\mathbf{1 3}$ & $3-\mathrm{NO}_{2}$ & 25.4 & $\mathbf{1 8}$ & $4-\mathrm{sec}-\mathrm{C}_{4} \mathrm{H}_{9}$ & 36.5 \\
$\mathbf{1 2}$ & $4-\mathrm{F}$ & 35.4 & $\mathbf{1 9}$ & $4-\mathrm{N}_{\left(-\mathrm{CH}_{3}\right)_{2}}$ & 28.8 \\
$\mathbf{9}$ & $4-\mathrm{Cl}$ & 33.2 & $\mathbf{6}$ & $3,4-\mathrm{Cl}_{2}$ & 19.8 \\
$\mathbf{1 0}$ & $4-\mathrm{CH}_{3}$ & 14.2 & $\mathbf{1 4}$ & $3,4-\left(\mathrm{CH}_{3}\right)_{2}$ & 26.7 \\
$\mathbf{8}$ & $4-\mathrm{OCH}_{3}$ & 30.8 & & & \\
\hline
\end{tabular}

\section{Experimental}

\section{General}

The melting points were determined on a Kofler block and are uncorrected. The samples for elemental analyses and biological tests were dried over $\mathrm{P}_{2} \mathrm{O}_{5}$ at $61^{\circ} \mathrm{C}$ and $66 \mathrm{~Pa}$ for $24 \mathrm{~h}$. Elemental analyses were performed on a C,H,N,S analyzer (FISONS AE 1110, Milano). The IR spectra were measured in $\mathrm{KBr}$ on a Nicolet Impact 400 apparatus. The purity of the compounds was checked by TLC, using petroleum ether:ethyl acetate $(1: 1)$ and chloroform:acetone (9:1) as the mobile phases. ${ }^{1} \mathrm{H}$ NMR spectra of new compounds were recorded for DMSO- $\mathrm{d}_{6}$ solutions at ambient temperature on a Varian Mercury-Vx BB 300 spectrometer (operating at $300 \mathrm{MHz}$ ). Chemical shifts were recorded as $\delta$ values in ppm, and were indirectly referenced to tetramethylsilane via the solvent signal $\left(2.49\right.$ for $\left.{ }^{1} \mathrm{H}\right)$.

Synthesis of alkanedioyl dichlorides

\section{Butanedioyl dichloride}

Phosphorus pentachloride $(0.328 \mathrm{~mol})$ was added to a finely powdered succinic acid $(0.400 \mathrm{~mol})$, and the mixture was heated at $120^{\circ} \mathrm{C}$ for 5 hours. Phosphorus oxychloride was then distilled off, and the crude product was purified by vacuum distillation (yield $75 \%$, b.p. $104-105^{\circ} \mathrm{C} / 3.33 \mathrm{kPa}$, lit. [17] b.p. $\left.103-104^{\circ} \mathrm{C} / 3.33 \mathrm{kPa}\right)$.

\section{Octanedioyl dichloride}

Suberic acid $(0.287 \mathrm{~mol})$ was heated with thionyl chloride $(1.260 \mathrm{~mol})$ at $120^{\circ} \mathrm{C}$ for 4 hours. After 
the removal of the excess reagent by distillation, the crude product was purified by distillation at reduced pressure (yield $69.20 \%$, b. p. $145^{\circ} \mathrm{C} / 1.33 \mathrm{kPa}$, lit. [18] b.p. $149-150^{\circ} \mathrm{C} / 1.60 \mathrm{kPa}$ ). The same protocol was used for the preparation of the other alkanedioyl dichlorides (yield; b.p.; b.p. [ref]): pentanedioyl dichloride $\left(90.7 \% ; 104^{\circ} \mathrm{C} / 2.53 \mathrm{kPa} ; 100^{\circ} \mathrm{C} / 2.00 \mathrm{kPa}\right.$ [19]); hexanedioyl dichloride (81.2\%; $\left.130-132^{\circ} \mathrm{C} / 2.40 \mathrm{kPa} ; 126^{\circ} \mathrm{C} / 1.60 \mathrm{kPa}[19]\right)$; nonanedioyl dichloride $\left(71.4 \% ; 162^{\circ} \mathrm{C} / 1.20 \mathrm{kPa} ; 158\right.$ $\left.159^{\circ} \mathrm{C} / 1.60 \mathrm{kPa}[20]\right)$.

Synthesis of N,N'-diarylalkanediamides

\section{$N, N$ '-Diarylethanediamides (1-4)}

Sodium ( $1 \mathrm{~g})$ was dissolved in absolute ethanol $(100 \mathrm{ml})$, a substituted aniline $(0.300 \mathrm{~mol})$ followed by diethyl oxalate $(0.150 \mathrm{~mol})$ were added to the solution, and the reaction mixture was heated at reflux for 1 hour. After cooling, the crude product was filtered off, washed with water, and crystallized from ethanol. The experimental data of compounds 1-4 are given in Table 1.

\section{Other N,N'-diarylalkanediamides (5-46)}

Butanedioyl dichloride $(0.016 \mathrm{~mol})$ was added dropwise to a stirred solution of an appropriate aniline $(0.032 \mathrm{~mol})$ in pyridine $(20 \mathrm{ml})$ at $0^{\circ} \mathrm{C}$. The reaction mixture was allowed to stand at ambient temperature for 24 hours, and then poured into water $(100 \mathrm{ml})$. The product was filtered off and crystallized from ethanol. The yields, melting points, IR and NMR spectral data as well as elemental analyses are summarized in Table 1 and Table 2.

\section{Biological assays}

\section{Antimycobacterial activity}

Antimycobacterial evaluation of $\mathrm{N}, \mathrm{N}^{\prime}$-diarylalkanediamides $(\mathrm{m}=0,3,4,6,7)$ was carried out in a semisynthetic liquid protein-containing Sula medium (IMUNA, Sarisske Michalany), buffered to $\mathrm{pH}=7.2$. The following mycobacterial strains were used: Mycobacterium tuberculosis $H_{37} R v, M$. kansasii PKG8, M. avium No. 80/72 and M. fortuitum 1021. The MICs were determined after 14 days of incubation at $37^{\circ} \mathrm{C}$. The compounds were added to the medium in dimethyl sulfoxide (DMSO) solutions. The final concentrations were $1000 ; 333 ; 111 ; 37 ; 12.3$; and $4.1 \mu \mathrm{mol} \mathrm{dm}{ }^{-3}$.

Antimycobacterial activity of N,N'-diarylbutanediamides $(\mathrm{m}=2)$ was determined in Sula semisynthetic medium (SEVAC, Prague). For evaluation of their in vitro antimycobacterial activity, the following strains were used: M. tuberculosis CNCTC My 1/47, M. kansasii CNCTC My 235/80, M. avium CNCTC My 80/72 and M. fortuitum CNCTC My 187/73 from the National Institute of Public Health, Prague. The compounds were added to the medium in DMSO solutions. The final concentra- 
tions were $1000 ; 500 ; 250 ; 125 ; 62 ; 31 ; 16 ; 8 ; 4 \mu \mathrm{mol} \mathrm{dm}{ }^{-3}$. The minimum inhibitory concentrations were determined after incubation at $37^{\circ} \mathrm{C}$ for 21 days.

MIC was the lowest concentration of a substance (on the above-stated concentration scale), at which inhibition of the growth of mycobacteria occurred. The compound is considered as active, when its MIC is lower than $1000 \mu \mathrm{mol} \mathrm{dm}{ }^{-3}$. DMSO concentration in the medium was maximum for concentration $1000 \mu \mathrm{mol} \mathrm{dm}{ }^{-3}$ of the tested compound and the control samples contained the same DMSO amount $(10 \mathrm{v} / \mathrm{v} \%)$. The applied DMSO content did not affect the antimycobacterial activity.

\section{Antialgal activity}

The inhibitory effect of selected N,N'-diarylalkanediamides on algal chlorophyll (Chl) production has been investigated in statically cultivated Chlorella vulgaris (96 hours; photoperiod $16 \mathrm{~h}$ light $/ 8 \mathrm{~h}$ dark; illumination: $5000 \mathrm{~lx} ; \mathrm{pH}=7.2$; Chl content at the beginning of cultivation: $0.5 \mathrm{mg} \mathrm{dm}^{-3}$ ) at room temperature and a constant inhibitor concentration $75 \mu \mathrm{mol} \mathrm{dm} \mathrm{dm}^{-3}$ according to Kralova et al. [21]. Chl content of algal suspensions was determined spectrophotometrically following its extraction into $\mathrm{N}, \mathrm{N}$-dimethylformamide according to Inskeep and Bloom [22]. The compounds were dissolved in DMSO as their solubility in water was insufficient. DMSO concentration in the algal suspensions did not exceed $0.75 \mathrm{v} / \mathrm{v} \%$ and the control samples contained the same DMSO amount as the suspensions treated with the tested compounds. The antialgal activity was expressed as the percentage of inhibition of the untreated control.

Acknowledgements: The authors wish to thank Mrs. J. Zizkova from the Department of Inorganic and Organic Chemistry for measurements of the IR spectra and Mrs. D. Karlickova from the Department of Pharmaceutical Chemistry and Drug Control, Faculty of Pharmacy, Charles University, for elemental analyses. This study was supported by the Ministry of Education of the Czech Republic (CEZ J 13/98: 11600001), and by the Scientific Grant Agency of the Ministry of Education of the Slovak Republic (grant No. 1/4013/97).

\section{References and Notes}

1. Waisser, K.; Odlerova, Z.; Grunert, R. Antituberkulotika. 43. Mitteilung. Eine neue gruppe potentieller Antituberkulotika - Oxalanilide und Malonanilide. Pharmazie 1989, 44, 162-163.

2. Dave, M. P.; Patel, J. M.; Langalia, N. A.; Shah, S. R.; Thaker, K. A. Studies on synthesis and antitubercular activity of some ( \pm )- $\alpha$-aceto-N,N'-diarylsuccinamide. J. Indian Chem. Soc. 1985, 62, 386-387.

3. Al-Arab, M. M.; Na'was, T. E.; Abu-Yousef, I. A. Synthesis of 2,3-diarylglutaramides and their antibacterial activity. Indian J. Chem. 1990, 29B, 694-696.

4. Stauffer Chemical Co.: Ger. Offen. 2451512 (1975).

5. Raynes, K.; Galatis, D.; Cowman, A. F.; Tilley, L.; Deady, L. W. Synthesis and activity of some antimalarial bisquinolines. J. Med. Chem. 1995, 38, 204-206. 
6. Kubicova, L.; Kadlcikova, J.; Waisser, K.; Kralova, K.; Sersen, F.; Slosarek, M.; Janota, J.; Svoboda, Z. Succinanilides and their photosynthesis-inhibiting and antimycobacterial activity. Folia Pharm. Univ. Carol. 1998, 23 (Suppl.), 148-149.

7. Kubicova, L.; Kralova, K.; Kunes, J.; Waisser, K. Synthesis of some N,N'-diphenylalkanediamides and their photosynthesis-inhibiting activity. Chem. Papers 2000, 54, 90-93.

8. Lapkin, I. I.; Andreichikov, Ju. S. Esters of heteroacids of the acetylene series. I. Addition of primary amines to esters of phenylethynylglyoxalic acid. Zh. Org. Khim. 1965, 1, 1212-1214.

9. Price, Ch. C.; Velzen, B. H. Some derivatives of oxanilide. J. Org. Chem. 1947, 12, 386-392.

10. CIBA-GEIGY A.-G.: U. S. 3906033 (1975); Chem. Abstr. 1976, 84, 4633 r.

11. Barnicoat, C. R. Melting points of the substituted amides of dibasic acids. J. Chem. Soc. 1927, 2926-2929.

12. Kirshanov, A. V.; Egorova, A. N. L. Fenilamidirovanie dvuchosnovnych karbonovych kislot difenilamidom. Zh. Obsch. Khim. 1953, 23, 1920-1922.

13. Ueda, M.; Morishima, M.; Kakuta, U.; Sugiyama, Y. Synthesis of ordered polyamides by direct polycondensation. Macromolecules 1992, 25, 6580-6585.

14. Sanna, A.; Repetto, G. Preparation of halodiphenylsuccinamides. Gazz. Chim. Ital. 1927, 57, 777780 .

15. Chen-Ku, L.; Chang-Shih, T.; Chi-Chieh, Ch. Synthesis of $\alpha, \omega$-bis(p-alkoxyphenylamino)alkanes and N,N'-bis(p-substituted phenyl)alkanedicarboxamides. Yao Hsueh Hsueh Pao 1957, 5, 333339. (ref. Chem. Abstr. 1961, 55, 25862i).

16. Hill, J. W.; Wallace, H. C. Studies of polymerization and ring formation. J. Amer. Chem. Soc. 1933, 55, 5023-5031.

17. Vorlaender, D. Über Aethylenester zweibasischer Säuren und Phenole. Justus Liebigs Ann. Chem. 1894, 280, 167-206.

18. Auwers, K.; Schmidt, M. Über die Konstitution der Chloride von 1,2- und 1,3-Dicarbonsäuren Chem. Ber. 1913, 46, 457-487.

19. Hoffmann, H. M. R., Haase, K.; Geschwinder, P. M. The synthesis of aliphatic dicarboxylic acid diiodides and aromatic analogues. Synthesis 1982, 3, 237-239.

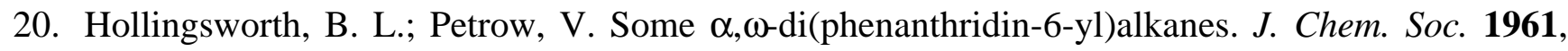
3664-3667.

21. Kralova, K.; Sersen, F.; Melnik, M. Inhibition of photosynthesis in Chlorella vulgaris by $\mathrm{Cu}(\mathrm{II})$ complexes with biologically active ligands. J. Trace Microprobe Techn. 1998, 16, 491-500.

22. Inskeep, W. P.; Bloom, P. R. Extinction coefficients of chlorophyll a and b in N,N-dimethylformamide and $80 \%$ acetone. Plant. Physiol. 1985, 77, 483-485.

Samples Availability: available from the authors.

(C) 2000 by MDPI (http://www.mdpi.org). Reproduction is permitted for noncommercial purposes. 